\title{
Increased Chlamydia Pneumoniae Igg after Coronary Artery Bypass Surgery
}

Teoman Zafer Apan ${ }^{1^{*}}$ and Alp Dolgun ${ }^{2}$

${ }^{1}$ Departmant of Medical Microbiology Kırıkkale University Faculty of Medicine, Kırıkkale, Turkey

2Department of Cardiovascular Surgery Yıldırım Bayezit Research and Training Hospital, Ankara, Turkey

"Corresponding author: Teoman Zafer Apan, Altınoluk Sokak Susurluk Sokak Y. İpek Apt No: 2 Daire:3 Aşağığlence/Etlik Keçiören, Ankara, Turkey, Tel: +90533 2235034; Fax: +903122522625; E-mail: teomanapan@yahoo.com

Rec date: Dec 03, 2014; Acc date: Jan 27, 2015; Pub date: Feb 05, 2015

Copyright: ( 2015 Apan TZ, et al. This is an open-access article distributed under the terms of the Creative Commons Attribution License, which permits unrestricted use, distribution, and reproduction in any medium, provided the original author and source are credited.

\begin{abstract}
Aim: The increasing body of evidence influences the possible association of Chlamydia pneumoniae infection with pathogenesis of atherosclerosis. The influence of coronary artery surgery on the disease activity is unclear. We aimed to determine the seroprevalence of $C$. pneumoniae before and after the coronary artery bypass grafting (CABG) surgery.
\end{abstract}

Methodology: One hundred and seventy eight patients diagnosed with coronary artery disease undergoing surgery were involved in the study. C. pneumoniae IgG and IgM seroprevalence were examined using ELISA method both the day before and 30 days after the surgery.

Results: IgG and IgM seroprevalence for C. pneumoniae were found to be $63.45 \%$ and $2.8 \%$ before the surgery. While there was significant increase on $\operatorname{lgG}(69.66 \%, p=0.001)$, increase was not significant on IgM values $(4.49 \%$, $p=0.250)$.

Conclusion: Our study showed that CABG surgery induced activation of chronic C. pneumoniae infection with surgical stress-induced immunological changes. Other possible factors, such as blood transfusion, are likely to be involved in this activation. Antimicrobial therapy during the operative period should be considered in patients who are serologically positive for C. pneumoniae after an operation.

Keywords: Coronary Artery Disease; Chlamydia Pneumoniae; Serology; Prevalence; Cardiovascular surgery

\section{Background}

Coronary artery disease is one of the most common causes of morbidity and mortality in the western community [1]. The progress of the disease is slow that makes difficult to define underlying etiopathogenesis. Hyperlipidemia, hypercholesterolemia, diabetes, cigarette smoking, genetic predisposition, and hypertension are accepted as common risk factors that determines only $50-60 \%$ of the patients. Studies performed in the recent years indicated the important role of the chronic infections in the progression of the disease [2,3].

C. pneumoniae, is an obligate intracellular microorganism that cause of upper respiratory tract infection. It has been considered as one of the infectious etiologies of atherosclerosis that first demonstrated by Saikku et al. [4]. The microorganism first appears in the lower respiratory tract and infected alveolar macrophages that transmigrate from the epithelium enter to the circulation and reach atherosclerotic plaque. C. pneumoniae may be capable of infecting the variety of cells including macrophages, vascular endothelial and smooth muscles. The presence of organism on atherosclerotic plaque has been shown by various techniques, including polymerase chain reaction, immunohystochemistry, and electron microscopy $[5,6]$.

Although the mechanisms are not clarified, the cell-mediated immunity is depressed especially with on pump cardiopulmonary bypass surgery [2,7]. Cardiac surgery has been also demonstrated to induce inflammatory cytokines and immune suppression [8]. Corticosteroid use during surgery further increased this effect [9]. Therefore, the possible change on serologic profile of disease is possible for the intracellular microorganism interacting with cell mediated immunity during surgery for the coronary artery disease that may be induced by various mechanisms [2]. The objective of the present study was to investigate the serologic profile of $C$. pneumoniae in the patients undergoing surgery for coronary artery bypass grafting (CABG) before, and after the surgery.

\section{Methods}

The study was performed under approval of Institutional Review Board and informed consents were obtained from each patient in Ministry of Health Ylldırım Beyazıt Hospital, Department of Cardiovascular Surgery March 2006 to October 2010. One hundred and seventy eight patients diagnosed with coronary artery disease undergoing CABG surgery were consecutively included to the study. As a part of their routine biochemical screening, venous blood samples withdrawn from patients before and $30^{\text {th }}$ day after the operation. To focus on just coronary artery disease and discus it on this field, the patients with rheumatoid valve or congenital heart disease, renal insufficiency, any type of malignancy, myocardial infarction within the past three months and unstable angina pectoris were excluded from the study. These patents were also rare to get them in to data. Patients suffered upper respiratory infection within 30 days after operation were excluded from the study. On-pump coronary surgery was performed under standard opioid-based anesthetic technique 
Page 2 of 4

including fentanyl, isoflurane, vecuronium bromide, using internal mammary artery or saphene vein graft. Prophylactic antimicrobial therapy (cefazolin $1 \mathrm{~g}$ i.v.) was given all patients during the preoperative period.

\section{ELISA}

Serum samples from the blood were separated and stored at minus $70^{\circ}$ until analyses. Investigator performing the ELISA was blinded about the origin of the samples including study or control patients. Commercially available kits (Euroimmun, Lübeck, Germany) that qualitatively screening the IgG and IgM class antibodies against $C$. pneumoniae were used. The serum samples where thawed at room temperature and diluted at $1 / 100$ for determining IgG and $1 / 10$ for $\operatorname{IgM}$, respectively. Controls and standards were performed using double-hole technique. Samples were remained about $20 \mathrm{~min}$ before investigating C. pneumoniae IgM for excluding the rheumatoid factors from the samples, and care was undertaken to obtain from the upper portions before distributing to the holes. All serum samples were incubated at room temperature for $30 \mathrm{~min}$. All holes were rinsed with $300 \mu \mathrm{L}$ solution using automatic machine (BIO-TEK ELx50). A specific enzyme conjugate $100 \mu \mathrm{L}$ was added to the each hole and incubated $30 \mathrm{~min}$ in room temperature. Samples were rinsed again with $300 \mu \mathrm{L}$ solution for three times and $100 \mu \mathrm{L}$ chromogen substrate was added and incubated in a dark at room temperature for $15 \mathrm{~min}$. A stop solution $100 \mu \mathrm{L}$ was added to the samples after incubation period and optic densities (At $450 \mathrm{~nm}$, ref $650 \mathrm{~nm}$ ) were determined using ELISA reader (SEAC Radim). The units corresponds the optic densities which were determined.

Results from C. pneumoniae IgG under $16 \mathrm{RU} / \mathrm{mL}$ were determined as negative and values exceeding $22 \mathrm{RU} / \mathrm{mL}$ were accepted as positive, and samples were reevaluated between these values. A cutoff value was determined for IgM and upper and lower values were accepted as positive and negative respectively. The values near the limit were again reevaluated.

\section{Statistics}

Data were analyzed using McNemar and Pearsons x2 tests using a package program (SPSS for Windows version15.0, SPSS Inc. Chicago, IL, USA). The relative risk values were determined after obtaining $95 \%$ confidence intervals. A p value was considered as 0.05 for statistical significance.

\section{Results}

One hundred and seventy eight patients (138 male, and 43 female), aged mean 56.24 years (ranging 24-74) were included to the study. The distribution of age and gender are shown in Table 1. All patients were undergone to the CABG using a classical technique. One coronary bypass or grafting was performed in 67 patients, two bypass required in 39 , three bypass was necessary in 39 patients, and left ventricular aneurysm repair was performed in 29 patients. 96 of 135 male patients (71.11\%) and 4 of 43 female patients (9.3\%) were smoking one pack year or more. Under 30 years of age no male or female patients were smoking.

\begin{tabular}{|l|l|l|l|l|}
\hline Age Interval & Male & Female & All & $\%$ \\
\hline $15-19$ & 0 & 0 & 0 & 0 \\
\hline $20-24$ & 1 & 1 & 2 & 1.1 \\
\hline
\end{tabular}

\begin{tabular}{|l|l|l|l|l|}
\hline $25-29$ & 0 & 0 & 0 & 0 \\
\hline $30-34$ & 1 & 1 & 2 & 1.1 \\
\hline $35-39$ & 7 & 1 & 8 & 4.5 \\
\hline $40-44$ & 9 & 3 & 12 & 6.7 \\
\hline $45-49$ & 13 & 6 & 19 & 10.7 \\
\hline $50-54$ & 24 & 6 & 30 & 16.9 \\
\hline $55-59$ & 31 & 6 & 37 & 20.8 \\
\hline $60-60+$ & 49 & 19 & 68 & 38.2 \\
\hline Total & 135 & 43 & 178 & 100 \\
\hline
\end{tabular}

Table 1: Distribution of age and gender.

There was significant difference on seropositivity for IgG anticor against $C$. pneumoniae between preoperative (63.45\%) and postoperative values $(69.66 \%, \mathrm{p}=0.001)$. On the other hand, a significant difference was not determined for IgM values including before $(2.8 \%)$ and after $(4.49 \%, \mathrm{p}=0.250)$ the surgery. Age related changes on C. pneumoniae IgG and IgM values are detailed on Table 2.

\begin{tabular}{|c|c|c|c|c|c|c|c|c|}
\hline Surgery & \multicolumn{4}{|c|}{ Before } & \multicolumn{4}{|c|}{ After } \\
\hline \multirow{2}{*}{ Age interval } & \multicolumn{2}{|l|}{$\lg G$} & \multicolumn{2}{|c|}{$\lg M$} & \multicolumn{2}{|l|}{$\lg G$} & \multicolumn{2}{|c|}{$\lg M$} \\
\hline & N & $\%$ & $\mathrm{~N}$ & $\%$ & $N$ & $\%$ & $N$ & $\%$ \\
\hline $15-19$ & 0 & 0 & 0 & 0 & 0 & 0 & 0 & 0 \\
\hline $20-24$ & 2 & 1.1 & 0 & 0 & 2 & 1.1 & 0 & 0 \\
\hline $25-29$ & 0 & 0 & 0 & 0 & 0 & 0 & 0 & 0 \\
\hline $30-34$ & 1 & 0.56 & 0 & 0 & 1 & 0.56 & 0 & 0 \\
\hline $35-39$ & 4 & 2.24 & 0 & 0 & 4 & 2.24 & 0 & 0 \\
\hline $40-44$ & 11 & 6.18 & 0 & 0 & 12 & 6.74 & 1 & 0.56 \\
\hline $45-49$ & 13 & 7.3 & 1 & 0.56 & 15 & 8.42 & 2 & 1.1 \\
\hline $50-54$ & 16 & 8.99 & 1 & 0.56 & 18 & 10.1 & 1 & 0.56 \\
\hline $55-59$ & 24 & 13.48 & 1 & 0.56 & 25 & 14.1 & 1 & 0.56 \\
\hline $60-60+$ & 42 & 23.6 & 2 & 1.1 & 47 & 26.4 & 3 & 1.68 \\
\hline Total & 113 & 63.45 & 5 & 2.8 & 124 & 69.7 & 8 & 4.49 \\
\hline
\end{tabular}

Table 2: Age-related change on C. pneumoniae IgG and IgM seropositivity.

\section{Discussion}

Atherosclerosis is the most important pathological process involved in coronary heart disease. Coronary artery disease is the most likely infectious etiology for patients undergoing surgery for CABG. The association between $C$. pneumoniae and coronary artery disease is well documented. However, little is known regarding $C$. pneumoniae seropositivity in patients undergoing CABG. C. pneumoniae cellular structures and genomic DNA segments have been directly observed in atherosclerotic vessels [9-11]. A recent study by Gürkan et al. found a 
significant correlation between increased aortic intimal thickness and C. pneumoniae seropositivity, although the exact cause was not determined [12]. The seroprevalence of $C$. pneumoniae is increased with advancing age, which could explain the higher incidence of $C$. pneumoniae in our study population. An even higher rate of $C$. pneumoniae was obtained in a recent study that investigated atherosclerotic patients from the same region. However, IgM values in this previous study are comparable with our results [13]. Our study showed a significant increase in $C$. pneumoniae IgG after CABG surgery compared with before CABG surgery, and a slight change in IgM values was observed after CABG surgery. Although this percentage change was small, it clearly demonstrates recent activity of the disease and the opportunistic nature of the microorganism. IgM is the first immunoglobulin to appear against $C$. pneumoniae that should be detected after 3 weeks. However, 6 to 8 weeks is required for an IgG response. To the best of our knowledge, this is the first report to find an increase in disease activity in the same population $[2,14]$.

Seropositivity may increase with contamination of blood or blood products with $C$. pneumoniae in the perioperative period. Approximately five units of blood or blood products were provided to every patient during CABG surgery during priming and on maintenance. Therefore, in the postoperative period, the seropositivity of $C$. pneumoniae may be increased, which may explain the results in our study. Donors can only give one unit in the limited time period. Therefore, five different persons' blood had to be provided (five units) to the same patient during surgery. $C$. pneumoniae seropositive transfusion can be performed in patients from donors that may result in a five-fold increase in seropositivity. In addition, if donors in our study had acute $C$. pneumoniae infection while giving blood to patients, this could also explain our finding of increased $C$. pneumoniae IgM seropositivity. Birkebaek et al. found IgG antibodies to $C$. pneumoniae in $42(40 \%)$ healthy blood donors and IgM antibodies to $C$. pneumoniae in one (1\%) healthy blood donor in their study [14]. Gencay et al. found IgG antibodies to $C$. pneumoniae in $64.3 \%$ of healthy adults, and this result may vary by location and time [15]. In a recent cohort study, antimicrobial therapies that were administered to Chlamydia-positive patients did not lead to symptomatic improvement in peripheral vascular disease [16]. Community-based eradication programs with antimicrobial therapy might not be effective in the dormant state of the microorganism. The presence of $C$. pneumoniae in atherosclerotic plaques, but its rare occurrence in the healthy vasculature, indicates that this microorganism might spread through dissemination from a primary infectious site with immune cells, namely $\mathrm{T}$ lymphocytes or monocytes [17]. This theory further supports the requirement of depletion of $\mathrm{T}$ cell function for activation and/or dissemination. Because of diversity in the population who are aged 15-35 years, specific age-related changes concerning $C$. pneumoniae seropositivity are difficult to determine.

Major surgery is able to induce immune suppression in the perioperative period by producing surgical stress, trauma, and preceding neuroendocrine responses, which affect $\mathrm{T}$ cell function and inflammatory mediators [18]. Anesthetics, including several intravenous agents, volatile anesthetics, and opioid use, as well as hypothermia, have also been demonstrated to contribute to immune suppression with decreasing $\mathrm{T}$ cell activity. The majority of these factors are mandatory in this type of operation [16]. In an in vitro study, opioids specifically inhibited $\mathrm{T}$ cell growth factor, other transcript activators, and $\mathrm{T}$ cell receptor-activated calcium flux in human T lymphocytes [2]. Whenever blood received from donors is indicated during surgery, administration to the patient may result in alterations in seropositivity.

The reasons for $C$. pneumoniae seroconversion, especially in patients undergoing CABG surgery, warrant further investigation. We speculate that increased inflammatory reactions or stunned immunity due to surgery may be major signals for bacterial reactivation. This requires continuous monitoring of the host's immune status by the microorganism that establishes its insidious nature, even in the dormant state. Heat shock proteins, which are increased in stressful circumstances, have a close homology with $C$. pneumoniae. Heat shock protein- 60 may also play a triggering role to activate this disease [18]. This may explain the diversity of the disease course or activity of C. pneumoniae between patients. Brunham and Rekart hypothesized that immunity is arrested by large-scale antimicrobial treatment to explain the increased rate of re-infection [16]. Another study showed C. pneumoniae antibodies in $79.7 \%$ of patients and $43.2 \%$ of controls. Additionally, elevated $C$. pneumoniae antibodies were also reported in male survivors of myocardial infarction [17]. We did not include a control group in our study because the primary aim of our study was to investigate the serological profile of $C$. pneumoniae in patients before and after surgery for CABG. In a study from our region, the control group had similar standards and there was a lower ratio (34\%) of $C$. pneumoniae IgG seropositivity than in patients [18]. C. pneumoniae infection, which is correlated with a chronic inflammatory response, can lead to atherosclerosis and other longterm clinical complications, such as myocardial infarction and stroke [17]. To prevent infectious complications, our clinical routine includes short-term, broad-spectrum antibiotherapy. Eradication of $C$. pneumoniae requires agent-specific antibiotic (azithromycin) treatment for at least 6 weeks [19]. Previous studies have shown that short-term therapy has no efficacy on progression of $C$. pneumoniaeinduced atherosclerosis [20].

Our clinic is a referral center for nearby cities and constitutes the majority of patients in our surgical population. However, patients were reluctant to be re-admitted for follow-up cardiac examinations, which were performed locally by cardiologists. Patients who were neglected or lost are possible reasons for loss of follow-up. Therefore, long-term follow-up was not possible, and is a limitation of the present study.

\section{Conclusion}

Our results suggest that coronary surgery increases $C$. pneumoniae IgG, and to a lesser extent, IgM seroprevalence. Increased $C$. pneumoniae IgG and IgM after CABG surgery not only depends on immunological mechanisms, but could also increase by blood transfusion from various donors. Therefore, antimicrobial therapy must be provided to all patients who are seropositive for $C$. pneumoniae at the postoperative period. Monitoring of increased seropositivity of $C$. pneumoniae IgM and IgG before and after CABG surgery could be an indicator for developing infection from blood donors. Prophylactic antibiotic therapy should be added to therapy for these patients to decrease their disease activity.

\section{References}

1. Ross R (1999) Atherosclerosis--an inflammatory disease. N Engl J Med 340: 115-126.

2. Mühldorfer I, Schafer KP, Emerging Bacterial pathogens Ed. Karger p: 65-68: 2001. 
Citation: Apan TZ, Dolgun A (2015) Increased Chlamydia Pneumoniae Igg after Coronary Artery Bypass Surgery . Clin Microbiol 4: 183. doi: $10.4172 / 2327-5073.1000183$

Page 4 of 4

3. Farsak B, Yildirir A, Akyön Y, Pinar A, Oç M, et al. (2000) Detection of Chlamydia pneumoniae and Helicobacter pylori DNA in human atherosclerotic plaques by PCR. J Clin Microbiol 38: 4408-4411.

4. Saikku P, Leinonen M, Mattila K, Ekman MR, Nieminen MS, et al. (1988) Serological evidence of an association of a novel Chlamydia, TWAR, with chronic coronary heart disease and acute myocardial infarction. Lancet 2 : 983-986.

5. Leinonen M, Saikku P (2002) Evidence for infectious agents in cardiovascular disease and atherosclerosis. Lancet Infect Dis 2: 11-17.

6. Campbell LA, Kuo CC (2004) Chlamydia pneumoniae--an infectious risk factor for atherosclerosis? Nat Rev Microbiol 2: 23-32.

7. Akbas H, Erdal AC, Demiralp E, Alp M (2002) Effects of coronary artery bypass grafting on cellular immunity with or without cardiopulmonary bypass: changes in lymphocytes subsets. Cardiovasc Surg 10: 586-589.

8. Rudensky B, Yinnon AM, Shutin O, Broide E, Wiener-Well Y, et al. (2010) The cellular immunological responses of patients undergoing coronary artery bypass grafting compared with those of patients undergoing valve replacement. Eur J Cardiothorac Surg 37: 1056-62.

9. Sano T, Morita S, Masuda M, Tomita Y, Nishida T, et al. (2003) Cardiopulmonary bypass, steroid administration, and surgical injury synergisticaaly impair memory $\mathrm{T}$ cell function and antigen presentation. Interact CardioVasc Thorac Surg 2: 598-602.

10. Kuo CC, Grayston JT, Campbell LA, Goo YA, Wissler RW, et al. (1995) Chlamydia pneumoniae (TWAR) in coronary arteries of young adults (15-34 years old). Proc Natl Acad Sci U S A 92: 6911-6914.

11. Maass M, Krause E, Engel PM, Krüger S (1997) Endovascular presence of Chlamydia pneumoniae in patients with hemodynamically effective carotid artery stenosis. Angiology 48: 699-706.

12. Gürkan S, Canbaz S, Gur O, Hüseyin S, Ege T, et al. (2013) The role of Chlamydia pneumoniae in the atherosclerotic process of patients under
50 years of age who underwent coronary artery bypass graft surgery. World J Cardiovasc Surg 3:146-151.

13. Kilic ZB, Poyraz O, Kilic AT (2012) Investigation of Chlamydophila pneumoniae seropositivity and risk factors in patients with atherosclerotic vascular disease in Sivas, Turkey. Mikrobiyol Bul 46: 156-158.

14. Birkebaek NH, Jensen JS, Seefeldt T, Degn J, Huniche B, et al. (2000) Chlamydia pneumoniae infection in adults with chronic cough compared with healthy blood donors. Eur Respir J 16: 108-111.

15. Gencay M, Dereli D, Ertem E, Serter D, Puolakkainen M, et al. (1998) Prevalence of Chlamydia pneumoniae specific antibodies in different clinical situations and healthy subjects in Izmir, Turkey. Eur J Epidemiol 14: 505-509.

16. Brunham RC, Rekart ML (2008) The arrested immunity hypothesis and the epidemiology of chlamydia control. Sex Transm Dis 35: 53-54.

17. Gupta S, Leatham EW, Carrington D, Mendall MA, Kaski JC, et al. (1997) Elevated Chlamydia pneumoniae antibodies, cardiovascular events, and azithromycin in male survivors of myocardial infarction. Circulation 96(2): 404-7.

18. Kaftan AH, Kaftan O (2000) Coronary artery disease and infection with chlamydia pneumonia. Jpn Heart J 41: 165-172.

19. Blasi F, Damato S, Cosentini R, Tarsia P, Raccanelli R, et al. (2002) Chlamydia InterAction with COPD (CIAC) study group. Chlamydia pneumoniae and chronic bronchitis association with severity and bacterial clearance following treatment. Thorax 5: 672-676.

20. Vainas T, Stassen FRM, Schurink GWH, Tordoir JHM, Welten RJThJ, et al. (2005) Secondary prevention of atherosclerosis through Chlamydia pneumoniae eradication (SPACE) trial: a randomized clinical trial in patients with peripheral arterial disease. Eur J Vasc Endovasc Surg 29:403-411. 